\title{
Aberrant expression of CDX2 is closely related to the intestinal metaplasia and MUC2 expression in intraductal papillary neoplasm of the liver in hepatolithiasis
}

\author{
Akira Ishikawa ${ }^{1,3}$, Motoko Sasaki ${ }^{1}$, Shusaku Ohira ${ }^{1,3}$, Tetsuo Ohta ${ }^{2}$, Koji Oda $^{3}$, Yuji \\ Nimura $^{3}$, Miin-Fu Chen ${ }^{4}$, Yi-Yin Jan ${ }^{4}$, Ta-Sen Yeh ${ }^{4}$ and Yasuni Nakanuma ${ }^{1}$ \\ ${ }^{1}$ Department of Human Pathology, Division of Surgical Oncology; ${ }^{2}$ Department of Gastrointestinal Surgery, \\ Kanazawa University Graduate School of Medicine, Kanazawa; ${ }^{3}$ Department of Surgery, Nagoya University \\ Graduate School of Medicine, Nagoya and ${ }^{4}$ Department of Surgery, Chang Gung Memorial Hospital, Tao
} Yuan, Taipei, Taiwan

\begin{abstract}
Intraductal papillary neoplasia of the liver (IPNL) frequently presents gastrointestinal metaplasia with aberrant expression of MUC2 and MUC5AC and oversecretion of mucin into the ductal lumen. In this study, the involvement of CDX2, a homeodomain protein involved in the regulation of intestinal development and differentiation, in the expression of MUC2 was examined in mucinous intrahepatic cholangiocarcinoma (ICC) $(n=7)$ and IPNL with hepatolithiasis $(n=19)$ with comparison to conventional ICC $(n=11)$, and intraductal papillary mucinous tumor and invasive ductal carcinoma of the pancreas $(n=9$ and 11 , respectively). A total of 33 cases of hepatolithiasis, extrahepatic biliary obstruction and normal livers were used as the control. Immunohistochemically, both MUC2 and MUC5AC were frequently expressed in mucinous ICC and IPNL, while expression of MUC2 was not seen in conventional ICC. The nuclear expression of CDX2 was closely associated with the expression of MUC2 in mucinous ICC and IPNL. This intimate association of MUC2 and CDX2 was confirmed by double immunostaining. The cytoplasmic CDX2 expression was frequent in the mucinous and the conventional ICC and pancreatic carcinoma, irrespective of MUC2 and MUC5AC expression. CDX2 mRNA was detected in neoplastic cells showing cytoplasmic as well as nuclear expression of CDX2 by reverse transcriptase-polymerase chain reaction. One IPMT expressed MUC2 associated with nuclear CDX2 expression, while the other IPMT and conventional pancreatic carcinoma expressed MUC5AC only. Aberrant expression of CDX2 is closely related to the overexpression of MUC2 in mucinous ICC and IPNL associated with hepatolithiasia, suggesting its role in intestinal differentiation and its association with carcinogenesis in these tumors.
\end{abstract}

Laboratory Investigation (2004) 84, 629-638, advance online publication, 29 March 2004; doi:10.1038/labinvest.3700087

Keywords: mucinous carcinoma; mucin-secreting tumor; cholangiocarcinoma; intestinal metaplasia; MUC2; MUC5AC; CDX2

Intrahepatic cholangiocarcinoma (ICC) is next in prevalence to hepatocellular carcinoma (HCC) among the primary liver carcinomas and shows a poor prognosis. Less than $10 \%$ of ICC patients have excessive mucin-producing tumor including mucinous carcinoma. Recently, intraductal mucin hypersecreting tumor resembling intraductal mucinous

Correspondence: Dr Y Nakanuma, MD, Department of Human Pathology, Kanazawa University Graduate School of Medicine, Kanazawa 920-8640, Japan.

E-mail: pbcpsc@kenroku.kanazawa-u.ac.jp

Received 6 October 2003; revised 3 January 2004; accepted 5 January 2004; published online 29 March 2004 papillary tumor of the pancreas (IPMT) has been studied and 'intraductal papillary neoplasia of the liver (IPNL)' was coined for such a condition. ${ }^{1}$ The prognosis of IPNL is rather favorable compared to conventional ICC. ${ }^{2,3}$ In these cases, dilated intrahepatic large and extrahepatic bile ducts are filled with papillary dysplastic or carcinomatous biliary epithelia with delicate fibrovascular stalks and oversecretion of mucin. Mucinous ICC is characteristically associated with some cases of IPNL. ${ }^{1}$ There are also mucin-producing carcinomas or neoplasm in the other exocrine organs such as the pancreas and breast, and these have a significantly favorable prognosis when compared to conventional 
carcinomas. ${ }^{4}$ These mucin-producing carcinomas share several histologic features, that is, mucinous ICC arising in hepatolithiasis is histologically characterized by well-circumscribed lakes of mucin that contain scant and detached malignant cells.

CDX2 is a caudal-related homeobox gene that encodes an intestine-specific transcription factor. ${ }^{5,6}$ CDX2 is expressed in intestinal epithelial cells of the goblet cell-type in normal colons. ${ }^{5,6}$ In addition, ectopic expression of CDX2 is known to occur in the intestinal metaplasia of the gastric mucosa ${ }^{7-10}$ and in Barrett's epithelium and inflammatory esophageal mucosa. ${ }^{11}$ Gastric carcinoma of the intestinal type and adenocarcinoma arising in Barrett's esophagus express CDX2.,8,11 In vitro experiments have revealed the significant functional effects of CDX genes on intestinal differentiation and the transcription of intestine-specific genes such as MUC2, liverintestine (LI) cadherin, and sucrase isomaltase..$^{5,6,12}$ The gastric mucosa of CDX2-expressing transgenic mice also express intestinal genes such as MUC2 and alkalinephosphatase, ${ }^{9}$ suggesting the CDX2dependent expression of these intestinal genes.

We reported previously that IPNL with and without mucinous ICC and chronic proliferative cholangitis associated with hepatolithiasis frequently present a gastric or intestinal differentiation characterized by the aberrant expression of MUC2 and MUC5AC, ${ }^{13,14}$ Given that the aberrant expression of CDX2 causes an intestinal differentiation with MUC2 expression in the stomach and esophagus, it is conceivable that CDX2 may be involved in the development of intestinal differentiation in the intrahepatic biliary tree in hepatolithiasis. ${ }^{13}$ However, it remains unclear whether or not the transcription of intestinal genes including MUC2 is regulated by CDX2 in IPNL and mucinous ICC. In this study, we examined the expression of CDX2 in IPNL arising in hepatolithiasis and conventional ICC, and also in conventional and mucin-secreting pancreatic carcinoma with respect to the expression of the MUC2 and MUC5AC mucins. Then, we comprehensively analyzed the significance of CDX2 and MUC2 in the development and progression of IPNL in hepatolithiasis.

\section{Materials and methods}

\section{Patients and Tissue Preparation}

Convetional carcinomas (11 cases of well/moderately differentiated ICC (adenocarcinoma), nine cases of well/moderately differentatied ductal invasive adenocarcinoma of the pancreas), and mucin oversecreting biliary and pancreatic neoplasms were used in this study. In the latter, seven cases of mucinous ICC associated with IPNL ${ }^{1}$ and nine cases of IPMT were included. All mucinous ICC with IPNL were associated with hepatolithiasis. IPNLs with mild-to-moderate dysplasia (IPNL1 and 2) ${ }^{1}$ $(n=19)$ were also evaluated.
All of these cases were surgically resected, and were obtained from our departments and affiliated hospitals. The main clinicopathological features of these cases are shown in Table 1. As controls with comparable age and sex distribution to the cases of IPNL, 19 cases of hepatolithiasis, eight cases of extrahepatic biliary obstruction, and six cases of histologically normal livers were used. All cases of hepatolithiasis alone or IPNL with hepatolithiasis showed chronic proliferative cholangitis in the nonneoplastic biliary parts. ${ }^{15}$ Three colorectal carcinomas were used as a known positive control for immunohistochemical detection of MUC2 and CDX2. The tissue specimens were fixed in $10 \%$ neutral formalin and embedded in paraffin. More than 20 thin sections, $4 \mu \mathrm{m}$ in thickness, were cut from each paraffin block, and several of them were processed for histological diagnosis and routine histological studies. The remaining were used for the following immunohistochemistry: formalinfixed and paraffin-embedded specimens from four cases each of mucinous ICC, conventional ICC, and IPNL associated with hepatolithiasis were used for the in situ hybridization study. In addition, three specimens of mucinous ICC, one of conventional colon carcinoma, four of conventional ICC, and two of normal control livers were used for the following reverse transcriptase-polymerase chain reaction (RTPCR), using microdissected tissues.

\section{Immunohistochemistry}

The distribution and expression of MUC2, MUC5AC and CDX2 were examined immunohistochemically. As for the primary antibodies, the following were used: MUC2 (Novocastra, New Castle, UK; close Ccp58, 1:100), MUC5AC (Novocastra, clone: CLH2, 1:500) and CDX2 (BioGenex, San Ramon, CA, USA; clone: CDX2-88, 1:100).

Table 1 Main clinicopathological features of the patients used in this study

\begin{tabular}{lrcc}
\hline Diseases & $\mathrm{n}$ & $\begin{array}{c}\text { Gender } \\
(M: F)\end{array}$ & $\begin{array}{c}\text { Age } \\
\text { (mean, range) } \\
\text { (years) }\end{array}$ \\
\hline Liver & & & \\
$\quad$ Mucinous carcinoma & 7 & $3: 4$ & $62.7(55-72)$ \\
Conventional ICC & 11 & $0: 11$ & $57.6(45-74)$ \\
IPNL & 19 & $11: 8$ & $51.1(34-83)$ \\
BECs in hepatolithiasis & 19 & $11: 8$ & $51.1(34-83)$ \\
BECs in EBO livers & 8 & $4: 4$ & $70.3(35-86)$ \\
$\quad$ BECs in normal livers & 6 & $2: 4$ & $42.3(29-57)$ \\
Pancreas & & & \\
$\quad$ IPMT & 9 & $0: 9$ & $66.3(55-74)$ \\
$\quad$ Conventional carcinoma & 11 & $6: 5$ & $61.6(33-77)$ \\
\end{tabular}

ICC, intrahepatic cholangiocarcinoma; conventional carcinoma, well or moderately differentiated adenocarcinoma; IPNL, intraductal papillary neoplasm of the liver; BECs, biliary epithelial cells; EBO, extrahepatic biliary obstruction; IPMT, intraductal papillary mucinous tumor. 
The deparaffinized and rehydrated tissue sections were pretreated in a microwave oven in citrate buffer $\left(\mathrm{pH} \mathrm{6)}\right.$ at $95^{\circ} \mathrm{C}$ for $20 \mathrm{~min}$ for antigen retrieval. Then, the sections were immersed in $0.3 \% \mathrm{H}_{2} \mathrm{O}_{2}$ in methanol for $20 \mathrm{~min}$ to abolish endogenous peroxidase activities, and immersed in 3\% normal goat serum diluted in phosphate-buffered saline for $60 \mathrm{~min}$. The sections were then incubated with one of the primary monoclonal antibodies described above at $4^{\circ} \mathrm{C}$ overnight. Then, the sections were treated with goat anti-rabbit immunoglobulins conjugated to peroxidase labeled-dextran polymer (Envision $+{ }^{\mathrm{TM}}$, Dako, Santa Barbara, CA, USA). The reaction products were visualized using 3-3'-diaminobenzidine tetrahydrochloride (Sigma Chemicals, St Louis, MO, USA) and $\mathrm{H}_{2} \mathrm{O}_{2}$. The sections were then lightly counterstained with hematoxylin. There was no positive staining when $\mathrm{H}_{2} \mathrm{O}_{2}$ without $\mathrm{DAB}$ or $\mathrm{DAB}$ without $\mathrm{H}_{2} \mathrm{O}_{2}$ was applied. Negative controls included substituting for the primary antibody with similarly diluted mouse control immunoglobulin G (Dako).

The immunohistochemical expression of MUC2, MUC5AC, and CDX2 was assessed as follows: in the conventional and mucin oversecreting carcinomas or neoplasms, MUC2 and MUC5AC were immunohistochemically expressed as a diffuse and fine granular staining pattern in their cytoplasm. In the goblet cells, goblet mucus itself was spared, although the cytoplasm surrounding the mucin globules was positive. As for CDX2, there were immunohistochemically two staining patterns in these carcinomas or neoplasms: nuclear immunoreactivity as well as a granular cytoplasmic staining pattern. These two stainings were mutually exclusive in individual cases. The neoplastic and carcinoma cells showed both staining patterns in a variable combination, while non-neoplastic colorectal mucosa showed only nuclear CDX2 expression. Both nuclear and cytoplasmic staining of CDX2 were evaluated separately with respect to the expression of MUC2 and MUC5AC in individual groups.

When more than $10 \%$ of carcinoma or neoplastic cells were positive for MUC2, MUC5AC, or CDX2, such cases were regarded as positive. Furthermore, when from 10 to $50 \%$ of carcinoma or neoplastic cells were positive, such cases were regarded as slightly positive, and when more than $50 \%$ of carcinoma or neoplastic cells were positive, such cases were regarded as markedly positive. In the biliary lining epithelia of the intrahepatic biliary tree of the control livers, the expression of MUC2, MUC5AC, and CDX2 was similarly evaluated.

\section{Double Immunostaining of CDX2 and MUC2, and of CDX2 and MUC5AC}

Double immunohistochemical staining was performed to assess simultaneous detection of CDX2 and MUC2, or CDX2 and MUC5AC, or MUC2 and MUC5AC in non-neoplastic and neoplastic biliary and carcinomatous cells. Two cases of IPNL, Two cases of conventional ICC, Two cases of mucinous colorectal carcinoma, one case of IPMT (in situ type), one case of conventional ductal pancreatic carcinoma, and two cases of hepatolithiasis alone were used for this study. That is, the deparaffinized sections were incubated with normal goat serum (diluted 1:10, Vector Laboratory, Burlingame, CA, USA) for $20 \mathrm{~min}$, immersed within $10 \mathrm{mM}$ citrate buffer and heated in a microwave oven at $95^{\circ} \mathrm{C}$ for $20 \mathrm{~min}$, and then added to mouse monoclonal antibody to CDX2 overnight at $4^{\circ} \mathrm{C}$, followed by a goat anti-mouse immunoglobulin conjugated to alkaline phosphatase labeled-dextran polymer (Dako Envision-AP ${ }^{\mathrm{TM}}$; neat; Dako) for $60 \mathrm{~min}$. Reaction product was visualized with a Vector $\operatorname{Red}^{\mathrm{TM}}$ Alkaline Phosphatase Substrate Kit I (Vector). After stopping color development, the sections were incubated in $10 \mathrm{mM}$ citrate buffer at $95^{\circ} \mathrm{C}$ for $20 \mathrm{~min}$ to inactivate the antigenicity of the first antibody, and then a mouse monoclonal anti-human antibody to MUC2 or MUC5AC as described above was applied overnight at $4^{\circ} \mathrm{C}$, followed by incubation with FITC-labeled goat anti-mouse immunoglobulin (diluted 1:40, Dako) for $60 \mathrm{~min}$. Then the sections were mounted using a Slow Fade ${ }^{\mathrm{TM}}$ Light Antifade Kit (Molecular Probes Inc., Eugene, OR, USA) and observed under fluorescent microscopy. No positive staining was obtained when primary monoclonal antibody was omitted or replaced by normal mouse serum in the negative controls of the staining procedures.

\section{RT-PCR of CDX2 and MUC2 mRNA using Samples Isolated from Microdissected Tissue}

A total RNA was isolated from three foci of mucinous ICC and a foci of conventional colorectal carcinoma with MUC2 and nuclear CDX2 expression, four foci of conventional ICC with cytoplasmic CDX2 and no MUC2, and two foci of normal intrahepatic large bile ducts expressing neither CDX2 nor MUC2 using a Pinpoint slide RNA isolation system II (Zymo Research, Orange, CA, USA). After cDNA was synthesized, RT-PCR was carried out using the intron-spanning PCR primer sets for the amplification of CDX2, MUC2, and GAPDH as follows: CDX2 forward, 5' -GGAACCTGTGCGAGTGG; reverse, 5'-TTCCTCCGGATGGTGATGTA (PCR products, $160 \mathrm{bp}$ ); MUC2 forward, 5'-CCCGGCCTCTGCGACTA; reverse, 5'-GCGGGT-GAGGTAGATGGTGT (PCR products, $156 \mathrm{bp}$ ); GAPDH forward, 5'-CGACAGTCA-GCCGCA-


products, $65 \mathrm{bp}$ ).

\section{Statistical Analysis}

Differences between each group were analyzed with Wilcoxon's rank sum test, and the significance level was defined as $P<0.05$. 


\section{Results}

\section{Nuclear and Cytoplasmic Expression of CDX2}

Two types of CDX2 expression pattern, nuclear (Figure 1a and g) and cytoplasmic (Figure 1d), were seen variously in carcinomas or non-neoplastic cells. The immunohistochemical expression pattern of CDX2 in each group is summarized in Table 2 and Figure 2. In the mucinous ICC, the nuclear expression of CDX2 was significantly frequent (in $71 \%$ of the cases), when compared with that of conventional ICC as well as IPMT, conventional pancreatic carcinoma. The cytoplasmic expression of CDX2 was seen in 29 and $64 \%$ of mucinous ICC and the conventional ICC, respectively. In all, 11\% of IPMT express nuclear CDX2, while none of the conventional pancreatic carcinoma expressed CDX2 in the nucleus. The cytoplasmic expression of CDX2 was seen in 33 and $73 \%$ of IPMT and conventional pancreatic carcinoma, respectively. Nuclear and cytoplasmic expression of CDX2 were seen focally in biliary epithelial cells (BECs) in IPNL (16 and $26 \%$, respectively), hepatolithiasis (16 and $26 \%$, respectively) and extrahepatic biliary obstruction (EBO) livers (25 and 13\%, respectively). CDX2 expression was not detected in any of the normal livers.

\section{Immunostaining of MUC2 and MUC5AC with Respect to CDX2 Expression}

In mucinous and the conventional biliary and pancreatic carcinomas, IPNL, and BECs in hepatolithiasis, MUC2 and MUC5AC were variously expressed in the cytoplasm of carcinomas and nonneoplastic epithelial cells as a diffuse staining pattern (Figure $1 \mathrm{~b}$ and $\mathrm{h}$. They tended to show clustering in some parts or areas of the carcinoma,
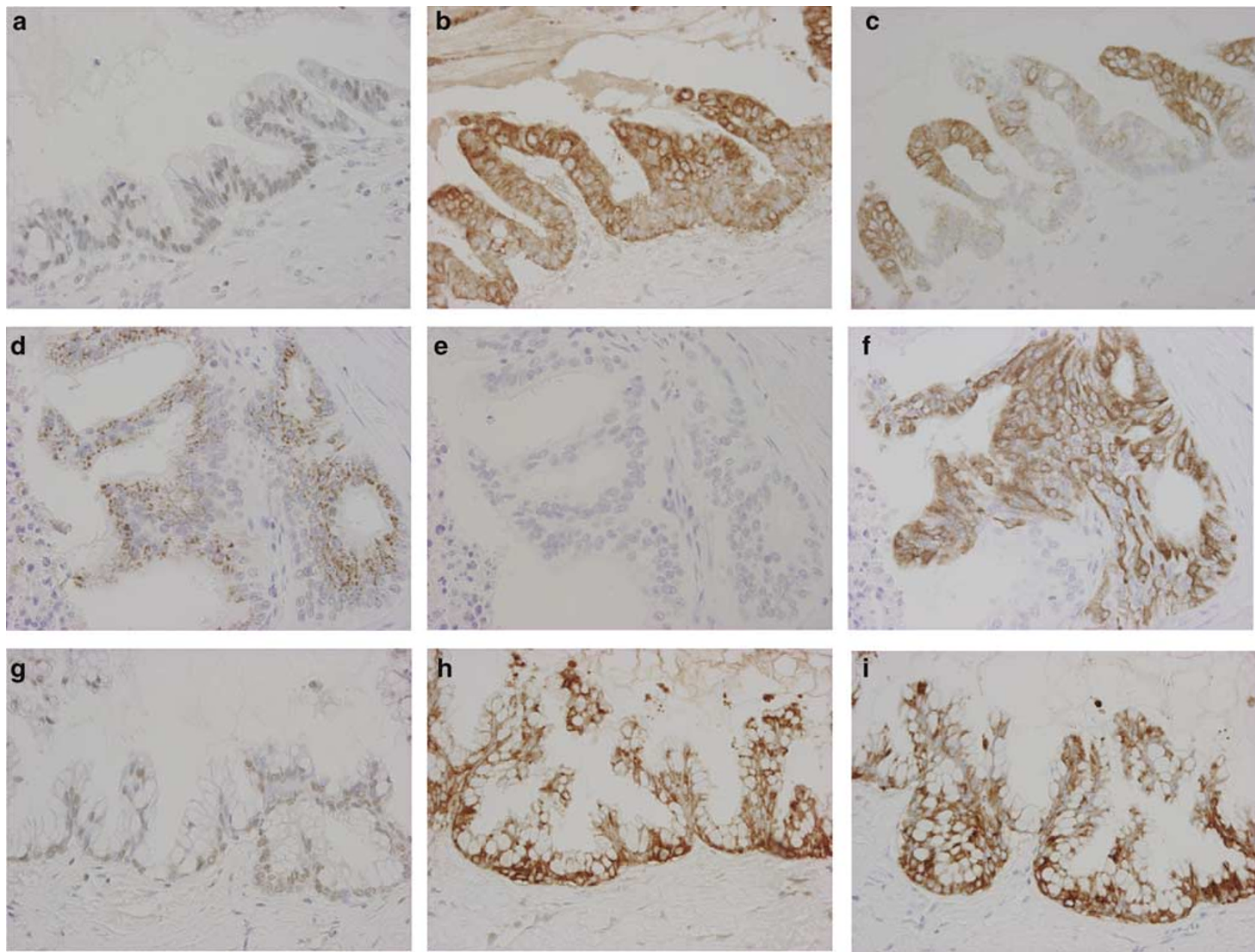

Figure 1 Expression of CDX2, MUC2, and MUC5AC in mucinous cholangiocarcinoma (a-c), in conventional pancreatic carcinoma (d-f), and in IPNL (g-i). (a) CDX2 was expressed in the nucleus of carcinoma cells. (b) MUC2 was expressed diffusely in the cytoplasm of carcinoma cells. (c) MUC5AC was expressed in the cytoplasm of carcinoma cells. (d) CDX2 was expressed in the supranuclear granules in the cytoplasm of carcinoma cells. (e) MUC2 was not expressed in the carcinoma cells. (f) MUC5AC was expressed in the cytoplasm of carcinoma cells. (g) CDX2 was expressed in the nucleus of cells of IPNL. (h) MUC2 is expressed diffusely in the cytoplasm of carcinoma cells. (i) MUC5AC was expressed extensively in the cytoplasm of carcinoma cells. Immunostaining for CDX2 (a, d, g), MUC2 (b, e, h), and MUC5AC (C, F, I) and hematoxylin. Original magnification, $\times 80$. 
Table 2 Expression of CDX2, MUC2, and MUC5AC

\begin{tabular}{|c|c|c|c|c|c|}
\hline \multirow[t]{2}{*}{ Diseases } & \multirow[t]{2}{*}{$\mathrm{N}$} & \multicolumn{2}{|c|}{$C D X 2$} & \multirow[t]{2}{*}{ MUC2 } & \multirow[t]{2}{*}{ MUC5AC (\%) } \\
\hline & & Nuclear (\%) & Cytoplasmic (\%) & & \\
\hline \multicolumn{6}{|l|}{ Liver } \\
\hline Mucinous carcinoma & 7 & $71^{\mathrm{a}, \mathrm{b}, \mathrm{d}}$ & 29 & $71^{\mathrm{a}, \mathrm{b}}$ & $86^{\mathrm{d}}$ \\
\hline Conventional ICC & 11 & 0 & $64^{\mathrm{c}, \mathrm{d}}$ & 0 & $91^{\mathrm{d}}$ \\
\hline IPNL & 19 & 16 & 26 & $32^{\mathrm{a}}$ & $100^{\mathrm{d}}$ \\
\hline BECs in hepatolithiasis & 19 & 16 & 26 & $42^{\mathrm{a}}$ & $100^{\mathrm{d}}$ \\
\hline BECs in EBO livers & 8 & 25 & 13 & 25 & 13 \\
\hline BECs in normal livers & 6 & 0 & 0 & 0 & 0 \\
\hline \multicolumn{6}{|l|}{ Pancreas } \\
\hline IPMT & 9 & 11 & 33 & 11 & $89^{\mathrm{d}}$ \\
\hline Conventional carcinoma & 11 & 0 & $73^{\mathrm{c}, \mathrm{d}}$ & 18 & $100^{\mathrm{d}}$ \\
\hline
\end{tabular}

ICC, intrahepatic cholangiocarcinoma; conventional carcinoma, well or moderately differentiated adenocarcinoma; IPNL, intraductal papillary neoplasm of the liver; BECs, biliary epithelial cells; EBO, extrahepatic biliary obstruction; IPMT, intraductal papillary mucinous tumor.

${ }^{\mathrm{a}} P<0.05$ vs IPMT and conventional pancreatic carcinoma.

${ }^{\mathrm{b}} P<0.05$ vs conventional ICC.

${ }^{\mathrm{c}} P<0.05$ vs IPNL and BECs in hepatolithiasis.

${ }^{\mathrm{d}} P<0.05$ vs BECs in EBO livers and normal livers.

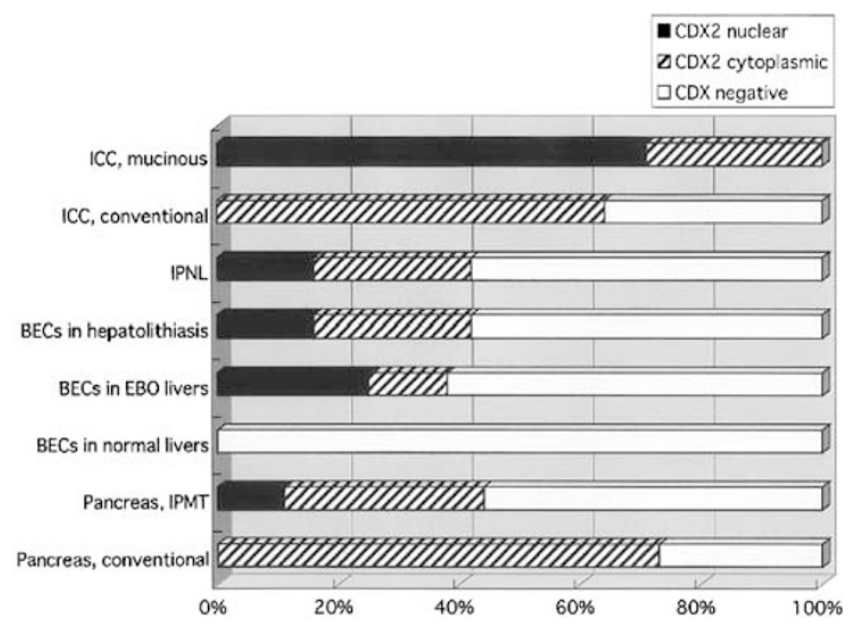

Figure 2 A graph showing CDX2 expression in mucinous and conventional carcinoma in intrahepatic bile ducts and the pancreas. ICC, intrahepatic cholangiocarcinoma; IPNL, intraductal papillary neoplasm of the liver; BECs, biliary epithelial cells; EBO, extrahepatic biliary obstruction; IPMT, intraductal papillary mucinous tumor.

although a number of positive cells were variable according to individual cases or places.

The expression of MUC2 was significantly frequent in mucinous ICC when compared with that in conventional ICC, IPMT, and conventional pancreatic carcinoma $(P<0.05)$ (Table 2$)$. In addition, MUC2 expression was increased in IPNL and BECs in hepatolithiasis when compared with that in BECs in EBO livers and normal livers $(P<0.05)$.

The correlation between MUC2 expression and the pattern of CDX2 expression is shown in Table 3. All of the five cases of mucinous ICC with nuclear CDX2 expression expressed MUC2, while none of the remaining mucinous ICC and conventional ICC cases, which lacked nuclear CDX2 expression, expressed MUC2 at all, irrespective of the cytoplasmic expression of CDX2. One case of IPMT with nuclear CDX2 expression expressed MUC2, whereas no cases of IPMT or conventional pancreatic carcinoma without nuclear CDX2 expressed MUC2 at all. In all of the cases of IPNL and hepatolithiasis with nuclear CDX2 expression, MUC2 was expressed. In addition, a focal expression of MUC2 was observed in IPNL and hepatolithiasis with cytoplasmic CDX2 expression. MUC2 expression in carcinoma and non-neoplastic cells was closely correlated to the nuclear expression of CDX2 $(P<0.05)$.

The expression of MUC5AC was significantly frequent in mucinous and the conventional ICC, IPMT, conventional pancreatic carcinoma, IPNL, and BECs in hepatolithiasis when compared with BECs in EBO livers (Table 2). The expression of MUC5AC was seen in the cytoplasm of carcinoma cells as well as non-neoplastic cells in hepatolithiasis (Figure 1c, f, and i). The correlation between MUC5AC expression and the pattern of CDX2 expression is shown in Table 4. There was no correlation between the expression of MUC5AC and the pattern of CDX2 expression.

In the surrounding pancreas, there was no expression of MUC2 or MUC5AC in the surrounding exocrine acini, ducts, or the islet cells in the cases of IPMT or conventional ductal carcinoma. In the surrounding liver around the mucinous ICC and IPNL with hepatolithiasis and hepatolithiasis alone of the control livers, MUC5AC and, to a lesser degree, MUC2 and CDX2 were expressed in the biliary epithelium of the intrahepatic biliary tree. However, such expressions were not evident in the EBO livers or in the surrounding non-neoplastic liver of conventional ICC cases. 
Table 3 Correlation between CDX2 expression pattern and MUC2 expression

\begin{tabular}{|c|c|c|c|c|c|c|c|}
\hline & & \multicolumn{2}{|c|}{ CDX2-nuclear } & \multicolumn{2}{|c|}{ CDX2-cytoplasmic } & \multicolumn{2}{|c|}{ CDX2-negative } \\
\hline & & $\operatorname{MUC2}(+)^{\mathrm{a}}$ & MUC2 (-) & MUC2 (+) & MUC2 (-) & MUC2 (+) & MUC2 (-) \\
\hline \multicolumn{8}{|l|}{ Liver } \\
\hline Mucinous carcinoma & 7 & $5 / 5$ & $0 / 5$ & $0 / 2$ & $2 / 2$ & NA & NA \\
\hline Conventional ICC & 11 & NA & NA & $0 / 7$ & $7 / 7$ & $0 / 4$ & $4 / 4$ \\
\hline IPNL & 19 & $3 / 3$ & $0 / 3$ & $3 / 5$ & $2 / 5$ & $2 / 11$ & $9 / 11$ \\
\hline BECs in hepatolithiasis & 19 & $3 / 3$ & $0 / 3$ & $3 / 5$ & $2 / 5$ & $0 / 11$ & $11 / 11$ \\
\hline BECs in EBO livers & 8 & $2 / 2$ & $0 / 2$ & $0 / 1$ & $1 / 1$ & $0 / 5$ & $5 / 5$ \\
\hline BECs in normal livers & 6 & NA & NA & NA & NA & $0 / 6$ & $6 / 6$ \\
\hline \multicolumn{8}{|l|}{ Pancreas } \\
\hline IPMT & 9 & $1 / 1$ & $0 / 1$ & $0 / 4$ & $4 / 4$ & $0 / 4$ & $4 / 4$ \\
\hline Conventional carcinoma & 11 & NA & NA & $1 / 8$ & $7 / 8$ & $1 / 3$ & $2 / 3$ \\
\hline Total & 84 & $14 / 14$ & $0 / 14$ & $7 / 32$ & $25 / 32$ & $3 / 44$ & $41 / 44$ \\
\hline
\end{tabular}

ICC, intrahepatic cholangiocarcinoma; conventional carcinoma, well or moderately differentiated adenocarcinoma; IPNL, intraductal papillary neoplasm of the liver; BECs, biliary epithelial cells; EBO, extrahepatic biliary obstruction; IPMT, intraductal papillary mucinous tumor.

${ }^{\mathrm{a}} \mathrm{P}<0.05$ vs MUC2 expression in CDX2-cytoplasmic and CDX2-negative groups.

Table 4 Correlation between CDX2 expression pattern and MUC5AC expression

\begin{tabular}{|c|c|c|c|c|c|c|c|}
\hline & & \multicolumn{2}{|c|}{ CDX2-nuclear } & \multicolumn{2}{|c|}{ CDX2-cytoplasmic } & \multicolumn{2}{|c|}{ CDX2-negative } \\
\hline & & $M U C 5 A C(+)$ & $\operatorname{MUC5AC}(-)$ & $M U C 5 A C(+)$ & MUC5AC (-) & MUC5AC(+) & $\operatorname{MUC5AC}(-)$ \\
\hline \multicolumn{8}{|l|}{ Liver } \\
\hline Mucinous carcinoma & 7 & $4 / 5$ & $1 / 5$ & $2 / 2$ & $0 / 2$ & NA & NA \\
\hline Conventional ICC & 11 & NA & NA & $6 / 7$ & $1 / 7$ & $4 / 4$ & $0 / 4$ \\
\hline IPNL & 19 & $3 / 3$ & $0 / 3$ & $5 / 5$ & $0 / 5$ & $11 / 11$ & $0 / 11$ \\
\hline BECs in hepatolithiasis & 19 & $3 / 3$ & $0 / 3$ & $5 / 5$ & $0 / 5$ & $11 / 11$ & $0 / 11$ \\
\hline BECs in EBO livers & 8 & $0 / 2$ & $2 / 2$ & $0 / 1$ & $1 / 1$ & $1 / 5$ & $4 / 5$ \\
\hline BECs in normal livers & 6 & NA & NA & NA & NA & $0 / 6$ & $6 / 6$ \\
\hline \multicolumn{8}{|l|}{ Pancreas } \\
\hline IPMT & 9 & $1 / 1$ & $0 / 1$ & $3 / 4$ & $1 / 4$ & $4 / 4$ & $0 / 4$ \\
\hline Conventional carcinoma & 11 & NA & NA & $8 / 8$ & $0 / 8$ & $3 / 3$ & $0 / 3$ \\
\hline Total & 84 & $11 / 14$ & $3 / 14$ & $29 / 32$ & $3 / 32$ & $34 / 44$ & $10 / 44$ \\
\hline
\end{tabular}

ICC, intrahepatic cholangiocarcinoma; conventional carcinoma, well or moderately differentiated adenocarcinoma; IPNL, intraductal papillary neoplasm of the liver; BECs, biliary epithelial cells; EBO, extrahepatic biliary obstruction; IPMT, intraductal papillary mucinous tumor.

\section{Double Immunostaining of CDX2 and MUC2, of CDX2 and MUC5AC, and of MUC2 and MUC5AC}

In the double immunostaining of CDX2 and MUC2, neoplastic and non-neoplastic biliary cells and carcinoma cells showed positive nuclear CDX2 and positive cytoplasmic MUC2, suggesting that CDX2 and MUC2 were simultaneously expressed in these cells (Figure 3a and b). While CDX2-positive cells were constantly positive for MUC2, a few MUC2positive cells were not positive for CDX2. MUC2positive cells outnumbered CDX2-positive cells. Neoplastic or carcinoma cells presenting with cytoplasmic CDX2 expression rarely expressed MUC2 in their cytoplasm. MUC2 was not detected in carcinoma, neoplastic, or non-neoplastic cells without nuclear CDX2 expression.

In the double immunostaining of CDX2 and MUC5AC, a majority of carcinoma cells with nuclear as well as cytoplasmic CDX2 expressed cytoplasmic MUC5AC. This was the case in biliary, pancreatic and colorectal carcinoma or mucinous neoplasm.

\section{RT-PCR for CDX2 mRNA and MUC2 mRNA}

CDX2 mRNA expression was detected in the tissue samples taken from MUC2-positive and nuclear CDX2-positive areas as well as in the samples taken from MUC2-negative and cytoplasmic CDX2-positive areas (Figure 4). CDX2 mRNA was not detected in the samples taken from MUC2-negative and CDX2-negative normal large bile ducts as a negative control (Figure 4). MUC2 mRNA was detected in all the tissue samples from MUC2-positive and nuclear CDX2-positive areas, and two of four samples taken from MUC2-negative and cytoplasmic CDX2-posi- 



Figure 3 Double staining of CDX2 and MUC2 in mucinous ICC (a) and conventional colorectal carcinoma as a positive control (b). CDX2 (red) was expressed in the nucleus and MUC2 (green) in the cytoplasm of carcinoma cells.

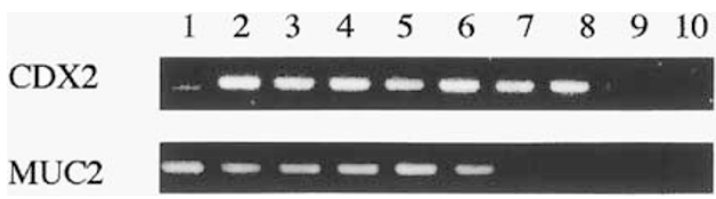

Figure 4 Expression of CDX2 and MUC2 mRNA detected by RTPCR in both cells with expression of nuclear and cytoplasmic CDX2 protein. A positive signal at $160 \mathrm{bp}$ for CDX2 mRNA was clearly detected in both samples from nuclear CDX2-positive areas (lanes 2-4) and cytoplasmic CDX2-positive areas (lanes 58). A weak positive signal is seen in lane 1 . A positive signal at $156 \mathrm{bp}$ for MUC2 mRNA was detected in samples from the nuclear CDX2-positive area (lanes 2-4) and two of four samples from the cytoplasmic CDX2-positive area (lanes 5-8). No signal was detected in the control samples without CDX2 and MUC2 expression (lanes 7 and 8). Lanes 1-4, samples from mucinous ICC (1-3) and conventional colorectal carcinoma (4) with nuclear CDX2 and MUC2 expression at the protein level; lanes 5 and 6, samples from conventional ICC with cytoplasmic CDX2 but not MUC2 expression at the protein level; lanes 7 and 8, samples from normal bile ducts without CDX2 and MUC2 expression.

tive areas (Figure 4). MUC2 mRNA was not detected in the samples taken from MUC2-negative and CDX2-negative normal large bile ducts as a negative control (Figure 4).

\section{Discussion}

BECs in intrahepatic large bile ducts express MUC3, but they do not express MUC2 and MUC5AC physiologically. ${ }^{14,16}$ In the present study, MUC5AC was expressed widely and MUC2 focally in reference cases of IPNL. Mucinous ICC associated with hepatolithiasis expressed both MUC2 and MUC5AC frequently. These findings agree with our previous study. ${ }^{13,17}$ In contrast to mucinous ICC, conventional ICC frequently expressed MUC5AC, but no MUC2, in carcinoma cells. Therefore, the expression of MUC2 seems to be a specific feature of mucinous ICC and IPNL.

Recent studies showed that CDX2, a regulator of the development, proliferation and differentiation of intestinal epithelial cells, ${ }^{5,12,18}$ is closely related to intestinal metaplasia in the gastric mucosa and
Barrett's esophagus. ${ }^{7-11,19}$ The present study clearly showed that MUC2 expression as an intestinal differentiation could be dependent on the nuclear expression of CDX2 in IPNL and mucinous ICC in the same manner as in the gastric mucosa and Barrett's esophagus. ${ }^{7-11,19}$ The CDX2 mRNA expression detected by RT-PCR using microdissected tissue confirmed the expression of CDX2 protein detected by immunohistochemistry. The expression of MUC2 in IPMT is also in parallel with the nuclear expression of CDX2. Two types of CDX2 expression, nuclear and cytoplasmic, were seen in the present study as described previously, ${ }^{8,12}$ and the nuclear expression of CDX2 was closely related to the MUC2 expression in IPNL, mucinous ICC, and some of IPMT. The nuclear expression of CDX2 seems to be more suitable as a transcriptional factor, which is responsible for intestinal differentiation and MUC2 expression in IPNL and other carcinomas..$^{5,12,18}$

The cytoplasmic pattern was reported in the stomach and Barrett's epithelium and in inflammatory esophageal mucosa. ${ }^{8,12}$ This pattern was correlated with neither MUC2 nor MUC5AC expression. The cytoplasmic expression of CDX2 as well as the nuclear type was not seen in the normal biliary epithelium. It is of interest that the present study showed that CDX2 mRNA was detected not only in the cells with nuclear CDX2 expression but also in the cells with cytoplasmic CDX2 expression by RTPCR using microdissected tissues. These findings confirm that the immunohistochemical cytoplasmic CDX2 expression was not caused by crossreaction of the antibody, and suggests that CDX2 protein is synthesized and retained in the cytoplasm. In the cells with cytoplasmic expression of CDX2, translocation of CDX2 protein synthesized in the cytoplasm might have been blocked, and therefore CDX2 protein accumulated in the cytoplasm and was detectable immunohistochemically. In the case of cyclin D1, which is also expressed in both the nucleus and cytoplasm, in vitro mutation of the cyclin D1 gene influences the localization of cyclin D1-cdk4 protein complexes to the cytoplasm in mouse cells. ${ }^{20,21}$ In addition, it has been suggested that cdk inhibitors, including p21, may have roles as 
adaptor proteins involved in the assembly and intracellular localization of the above proteins. ${ }^{21}$ Therefore, it is likely that an additional unknown factor such as an adaptor or inhibitor may be involved in the localization of CDX2. It remains to be clarified what kind of factor is critical for the regulation of CDX2 translocation. It is unknown why MUC2 mRNA was detected by RT-PCR in 2 of 4 RNA samples taken from the cytoplasmic CDX2positive areas in spite of negative immunohistochemical expression of MUC2. Since RT-PCR is such a sensitive method for the detection of a small amount of mRNA, it is plausible that a very low level of MUC2 mRNA, which is not enough to make immunohistochemical MUC2 expression, may be detected in the present study.

Intestinal-type gastric cancer is often preceded by intestinal metaplasia in humans. The genetic events responsible for the transdifferentiation that occurs in intestinal metaplasia are not well understood. CDX2 is a key molecule that causes intestinal metaplasia and the subsequent carcinoma of the intestinal type. ${ }^{7-11}$ Similarly, CDX2-dependent intestinal metaplasia is noted in Barrett's esophagus, which is followed by esophageal adenocarcinoma. ${ }^{11}$ The present study also showed CDX2-dependent intestinal metaplasia in IPNL, which is proposed as a premalignant lesion in intrahepatic biliary tree. Therefore, CDX2 is a common key molecule in IPNL, gastric carcinoma, and Barrett's adenocarcinoma, in which transient or constant intestinal metaplasia is important in the carcinogenesis. So, it is conceivable that a common factor, which promotes or regulates CDX2 expression, is closely related to the pathway of stepwise carcinogenesis of these carcinomas.

There have only been a few reports regarding the factors that regulate CDX2 expression. ${ }^{22-26}$ Chronic acid exposure ${ }^{25}$ and butyrate ${ }^{23}$ induce the expression of CDX2, and phosphatase and tensin homolog deleted from chromosome 10 (PTEN) stimulate CDX2 protein expression, ${ }^{22}$ reportedly. Inversely, a low concentration of TNF- $\alpha,{ }^{22}$ and oncogenic ras activation, ${ }^{26}$ decrease CDX2 expression. Since bacterial infection is usually associated with hepatolithiasis, it is plausible that butyrate or other acidic biproducts of fermentation by bacteria may be involved in the aberrant expression of CDX2 in BESs and IPNL in hepatolithiasis. The participation of TNF- $\alpha$ in the regulation of CDX2 expression needs to be elucidated, since our group recently reported the protein kinase C-dependent induction of MUC2 and MUC5AC by TNF- $\alpha$ in cultured murine BECs. ${ }^{27}$

In colorectal, pancreatic, and biliary tumors, the expression of two well-characterized mucin antigens, MUC1 and MUC2, has been correlated with the aggressiveness of these tumors. MUC1 expression was more frequent in invasive conventional carcinoma with poor prognosis, whereas MUC2 expression was higher in the mucinous type of these tumors with a favorable outcome. ${ }^{28-31}$ MUC2 itself has a property of a tumor suppressor gene. ${ }^{32}$
Furthermore, accumulating data suggest that CDX2 functions as a tumor suppressor. Forced expression of CDX2 in various intestinal epithelial cell lines inhibits cell proliferation and stimulates cell differentiation and apoptosis. ${ }^{26,33}$ CDX2 restoration in CDX2-negative cells decreased the proliferative activity. ${ }^{34}$ CDX2 expression decreases with the tumor grade in human colon cancers and in chemically induced tumors in the rat. ${ }^{35}$ In fact, we have reported that the proliferative activity is lower in MUC2-positive, suspected CDX2-positive gallbladder carcinoma when compared with MUC2negative carcinoma cells. ${ }^{36}$ Taking these facts into consideration, CDX2-dependent regulation of cell proliferation may be an important factor in defining the prognosis of the patient.

The present study shows that MUC5AC, which implies a gastric foveolar metaplasia, is aberrantly expressed in IPNL and conventional ICC, and also in IPMT and conventional ductal pancreatic carcinoma. We have reported that aberrant expression of MUC5AC frequently occurs in the biliary mucosa in hepatolithiasis, and biliary epithelial dysplasia and IPNL. ${ }^{14,17,37}$ The aberrant expression of MUC5AC has also reportedly been seen in the early step of pancreatic carcinoma. ${ }^{38}$ Therefore, aberrant expression of MUC5AC may be a common feature suggesting the early step of carcinogenesis in epithelial cells of intrahepatic bile ducts and pancreatic ducts. Although cytoplasmic CDX2 was frequently observed in the same diseases in which MUC5AC expression was evident, there was no significant correlation between the expression of cytoplasmic CDX2 and that of MUC5AC. At the moment, we have no evidence suggesting which molecules are involved in the transcription of gastric metaplasia and the aberrant expression of MUC5AC.

Mucin-secreting tumors of the intrahepatic biliary tract show frequent expression of MUC2 and nuclear CDX2. MUC2 expression as an intestinal differentiation is closely related to the expression of CDX2 in IPNL and mucinous ICC associated with hepatolithiasis in the same manner as intestinal metaplasia in gastric mucosa and Barrett's esophagus. This suggests that a common pathway of carcinogenesis may be involved in ICC associated with hepatolithiasis, gastric carcinoma, and adenocarcinoma in Barrett's esophagus, in which CDX2-dependent intestinal metaplasia develops.

\section{References}

1 Chen T, Nakanuma Y, Zen Y, et al. Intraductal papillary neoplasm of the liver associated with hepatolithiasis. Hepatology 2001;34:651-658.

2 Nakanuma Y, Sasaki M, Ishikawa A, et al. Biliary papillary neoplasm of the liver. Histol Histopathol 2002;17:851-861.

3 Chen MF, Jan YY, Chen TC. Clinical studies of mucinproducing cholangiocellular carcinoma: a study of 22 
histopathology-proven cases. Ann Surg 1998;227: 63-69.

4 Adsay NV, Merati K, Nassar H, et al. Pathogenesis of colloid (pure mucinous) carcinoma of exocrine organs: coupling of gel-forming mucin (MUC2) production with altered cell polarity and abnormal cell-stroma interaction may be the key factor in the morphogenesis and indolent behavior of colloid carcinoma in the breast and pancreas. Am J Surg Pathol 2003;27: 571-578.

5 Werling RW, Yaziji H, Bacchi CE, et al. CDX2, a highly sensitive and specific marker of adenocarcinomas of intestinal origin: an immunohistochemical survey of 476 primary and metastatic carcinomas. Am J Surg Pathol 2003;27:303-310.

6 Yamamoto H, Bai YQ, Yuasa Y. Homeodomain protein CDX2 regulates goblet-specific MUC2 gene expression. Biochem Biophys Res Commun 2003;300:813-818.

7 Almeida R, Silva E, Santos-Silva F, et al. Expression of intestine-specific transcription factors, CDX1 and CDX2, in intestinal metaplasia and gastric carcinomas. J Pathol 2003;199:36-40.

8 Bai YQ, Yamamoto H, Akiyama Y, et al. Ectopic expression of homeodomain protein CDX2 in intestinal metaplasia and carcinomas of the stomach. Cancer Lett 2002;176:47-55

9 Silberg DG, Sullivan J, Kang E, et al. Cdx2 ectopic expression induces gastric intestinal metaplasia in transgenic mice. Gastroenterology 2002;122:689-696.

10 Mutoh H, Hakamata Y, Sato K, et al. Conversion of gastric mucosa to intestinal metaplasia in Cdx2expressing transgenic mice. Biochem Biophys Res Commun 2002;294:470-479.

11 Eda A, Osawa H, Satoh K, et al. Aberrant expression of CDX2 in Barrett's epithelium and inflammatory esophageal mucosa. J Gastroenterol 2003;38:14-22.

12 Hinoi T, Lucas PC, Kuick R, et al. CDX2 regulates liver intestine-cadherin expression in normal and malignant colon epithelium and intestinal metaplasia. Gastroenterology 2002;123:1565-1577.

13 Shimonishi T, Zen Y, Chen TC, et al. Increasing expression of gastrointestinal phenotypes and p53 along with histologic progression of intraductal papillary neoplasia of the liver. Hum Pathol 2002;33: 503-511.

14 Sasaki M, Nakanuma Y, Kim Y. Expression of apomucins in the intrahepatic biliary tree in hepatolithiasis differs from that in normal liver and extrahepatic biliary obstruction. Hepatol 1998;27:46-53.

15 Nakanuma Y, Yamaguchi K, Ohta G, et al. Pathologic features of hepatolithiasis in Japan. Hum Pathol 1988;19:1181-1186.

16 Sasaki M, Nakanuma Y, Terada T, et al. Biliary epithelial expression of MUC1, MUC2, MUC3 and MUC5/6 apomucins during intrahepatic bile duct development and maturation. An immunohistochemical study. Am J Pathol 1995;147:574-579.

17 Sasaki M, Nakanuma Y, Kim Y. Characterization of apomicin expression in intrahepatic cholangio-carcinomas and their precursor lesions: an immunohistochemical study. Hepatology 1996;24:1074-1078.

18 Qualtrough D, Hinoi T, Fearon E, et al. Expression of CDX2 in normal and neoplastic human colon tissue and during differentiation of an in vitro model system. Gut 2002;51:184-190.

19 Eda A, Osawa H, Yanaka I, et al. Expression of homeobox gene CDX2 precedes that of CDX1 during the progression of intestinal metaplasia. J Gastroenterol 2002;37:94-100.

20 Diehl JA, Sherr CJ. A dominant-negative cyclin D1 mutant prevents nuclear import of cyclindependent kinase 4 (CDK4) and its phosphorylation by CDK-activating kinase. Mol Cell Biol 1997;17: 7362-7374.

21 Dhar KK, Branigan K, Parkes J, et al. Expression and subcellular localization of cyclin D1 protein in epithelial ovarian tumour cells. $\mathrm{Br} \mathrm{J}$ Cancer 1999;81:1174-1181.

22 Kim S, Domon-Dell C, Wang Q, et al. PTEN and TNFalpha regulation of the intestinal-specific $\mathrm{Cdx}-2$ homeobox gene through a PI3 K, PKB/Akt, and NFkappaB-dependent pathway. Gastroenterology 2002;123:1163-1178.

23 Domon-Dell C, Wang Q, Kim S, et al. Stimulation of the intestinal $\mathrm{Cdx} 2$ homeobox gene by butyrate in colon cancer cells. Gut 2002;50:525-529.

24 Rings EH, Boudreau F, Taylor JK, et al. Phosphorylation of the serine 60 residue within the $\mathrm{Cdx} 2$ activation domain mediates its transactivation capacity. Gastroenterology 2001;121:1437-1450.

25 Marchetti M, Caliot E, Pringault E. Chronic acid exposure leads to activation of the cdx2 intestinal homeobox gene in a long-term culture of mouse esophageal keratinocytes. J Cell Sci 2003;116: 1429-1436.

26 Lorentz O, Cadoret A, Duluc I, et al. Downregulation of the colon tumour-suppressor homeobox gene Cdx-2 by oncogenic ras. Oncogene 1999;18:87-92.

27 Zen Y, Harada K, Sasaki M, et al. Lipopolysaccharide induces overexpression of MUC2 and MUC5AC in cultured biliary epithelial cells: possible key phenomenon of hepatolithiasis. Am J Pathol 2002;161: 1475-1484.

28 Higashi M, Yonezawa S, Ho JJ, et al. Expression of MUC1 and MUC2 mucin antigens in intrahepatic bile duct tumors: its relationship with a new morphological classification of cholangiocarcinoma. Hepatology 1999;30:1347-1355.

29 Yamashita K, Yonezawa S, Tanaka S, et al. Immunohistochemical study of mucin carbohydrates and core proteins in hepatolithiasis and cholangiocarcinoma. Int J Cancer 1993;55:82-91.

30 Amaya S, Sasaki M, Watanabe Y, et al. Expression of MUC1 and MUC2 and carbohydrate antigen Tn change during malignant transformation of biliary papillomatosis. Histopathology 2001;38:550-560.

31 Terris B, Dubois S, Buisine MP, et al. Mucin gene expression in intraductal papillary-mucinous pancreatic tumours and related lesions. J Pathol 2002;197: 632-637.

32 Velcich A, Yang W, Heyer J, et al. Colorectal cancer in mice genetically deficient in the mucin Muc2. Science 2002;295:1726-1729.

33 Mallo GV, Soubeyran P, Lissitzky JC, et al. Expression of the $\mathrm{Cdx} 1$ and $\mathrm{Cdx} 2$ homeotic genes leads to reduced malignancy in colon cancer-derived cells. J Biol Chem 1998;273:14030-14036.

34 Hinoi T, Loda M, Fearon ER. Silencing of CDX2 expression in colon cancer via a dominant repression pathway. J Biol Chem 2003;278:44608-44616.

35 Ee HC, Erler T, Bhathal PS, et al. Cdx-2 homeodomain protein expression in human and rat colorectal adenoma and carcinoma. Am J Pathol 1995;147: $586-592$. 
36 Yamato T, Sasaki M, Watanabe Y, et al. Expression of MUC1 and MUC2 mucin core proteins and their messenger RNA in gall bladder carcinoma: an immunohistochemical and in situ hybridization study. J Pathol 1999;188:30-37.

37 Sasaki M, Tsuneyama K, Nakanuma Y. Aberrant expression of trefoil factor family-1 in biliary epithe- lium in hepatolithiasis and cholangiocarcinoma. Lab Invest 2003;83:1403-1414.

38 Kim GE, Bae HI, Park HU, et al. Aberrant expression of MUC5AC and MUC6 gastric mucins and sialyl Tn antigen in intraepithelial neoplasms of the pancreas. Gastroenterology 2002;123: 1052-1560. 